\title{
Characterization of Ferroelectric Phase Transition in Deuterated Glycine Phosphite Crystal
}

\author{
J. Furtak, S. Dacko and Z. Czapla \\ Institute of Experimental Physics, University of Wrocław \\ M. Borna 9, 50-204 Wrocław, Poland
}

(Received June 3, 2003)

\begin{abstract}
Dielectric, dilatometric, and ultrasonic studies around the ferroelectric phase transition at $319 \mathrm{~K}$ were done. Measurement of relative electric permittivity showed the existence of a strong bias field which causes in studied samples some diffused character of phase transition. However, all presented measurement confirmed the continuous character of phase transition. The obtained data are similar to those received earlier in the case of hydrogenated glycine phosphite crystal.
\end{abstract}

PACS numbers: $77.80 . \mathrm{Bh}$

\section{Introduction}

Glycine phosphite (GPI) is an interesting crystal exhibiting ferroelectric phase transition at $223 \mathrm{~K}[1,2]$. So far numerous studies including structural, ultrasonic, optical, dielectric dispersion, and electric field influence on the crystal have been done [3-7]. At room temperature the GPI belongs to the space group $P 2_{1} / a$. Deuterated crystal (D-GPI) is ferroelectric at room temperature and it belongs to the space group $P 2_{1}$. In the GPI crystal a strong isotope effect was observed and the shift of phase transition temperature upon deuteration was equal to $97 \mathrm{~K}[8,9]$. Thus, the molecular mechanism of phase transition is closely related to proton (deuteron) ordering along the $c$-axis which produces noncompensated dipole moment along the ferroelectric $b$-axis. This dipole moment originates from distortion of $\mathrm{PO}_{3} \mathrm{H}$ groups. Because of lack of experimental data related to characterization of the phase transition in deuterated crystal we decided to study physical properties of D-GPI crystal by means of dielectric, dilatometric, and ultrasonic methods. 


\section{Experimental}

D-GPI polycrystals were obtained by threefold recrystallization of hydrogenated polycrystals of GPI from $\mathrm{D}_{2} \mathrm{O}$. Single crystals were grown from saturated solution of deuterated polycrystals dissolved in $\mathrm{D}_{2} \mathrm{O}$ at constant temperature of $303 \mathrm{~K}$ by slow evaporation method. Dielectric and pyroelectric measurements were performed along the crystallographic $b$-axis (ferroelectric one) in order to check quality of the crystal and to find coercive field and existence of internal bias field $E_{\mathrm{b}}$. The sizes of the used samples were $1 \times 4 \times 4 \mathrm{~mm}^{3}$. The samples with the length about $5 \mathrm{~mm}$ cut along the $a, b$, and $c^{*}\left(c^{*}=c \sin \beta\right)$, crystallographic directions were prepared for crystal thermal expansion and ultrasonic measurement. Thermal expansion was studied by means of quartz capacitance dilatometer. Capacitance was measured using HP 4184 A RLC meter at the frequency of $1 \mathrm{kHz}$ both for dielectric and dilatometric measurement. The constant rate of temperature changes was equal to $0.5 \mathrm{~K} / \mathrm{min}$ and $0.1 \mathrm{~K} / \mathrm{min}$ in dielectric and dilatometric measurements, respectively. Dielectric and dilatometric measurements were done in the temperature range $290-340 \mathrm{~K}$ on heating run. Ultrasonic measurements were carried out using continuous wave-echo overlap method [10] with an accuracy of the order of $10^{-4}-10^{-5}$. The accuracy of the absolute velocity determination was about $1 \%$. The acoustic waves were excited by $\mathrm{LiNbO}_{3}$ transducer with resonance frequency $f=10 \mathrm{MHz}$ and band width $\Delta f=0.1 \mathrm{MHz}$. The ultrasonic wave propagation was studied along the $a, b$, and $c^{*}$ axes in temperature range of $290-340 \mathrm{~K}$. The rate of temperature changes was about $0.1 \mathrm{~K} / \mathrm{min}$.

\section{Results and discussion}

The temperature dependence of the dielectric permittivity of the D-GPI samples is shown in Fig. 1a. The sharp maximum of relative electric permittivity observed at $319 \mathrm{~K}$ showed that degree of deuteration is very high. It is in agreement with previous observed $T_{\mathrm{c}}$ in deuterated crystals [8,9]. The maximum value of the $\varepsilon$ is not so high and this is related to the existence of internal bias field $\left(E_{\mathrm{b}}\right)$. From hysteresis loop observation (Fig. 1b) we found $E_{\mathrm{b}}$ equal to $122 \mathrm{kV} / \mathrm{m}$ at room temperature. Existence of $E_{\mathrm{b}}$ is very typical in the case of deuterated crystals and we could not find any samples free of internal bias field.

The temperature dependence of $P_{\mathrm{s}}$ obtained from pyroelectric measurement is characteristic of unipolar (monodomain) state (Fig. 1).

Temperature dependences of relative linear expansion $\Delta L_{i} / L_{i}$ along three crystallographic axes are shown in Fig. 2.

No sharp anomalies of relative elongation of the samples $\Delta L_{i} / L_{i}$ were observed along any crystallographic directions at $T_{\mathrm{c}}$. Starting from $290 \mathrm{~K}$ nearly linear increase in elongation is observed along the $a$-axis up to $312 \mathrm{~K}$ and next we deal with a continuous and round change of relative elongation. At about $320 \mathrm{~K}$ relative sample elongation is again linear and its increase is rather weak. On the 

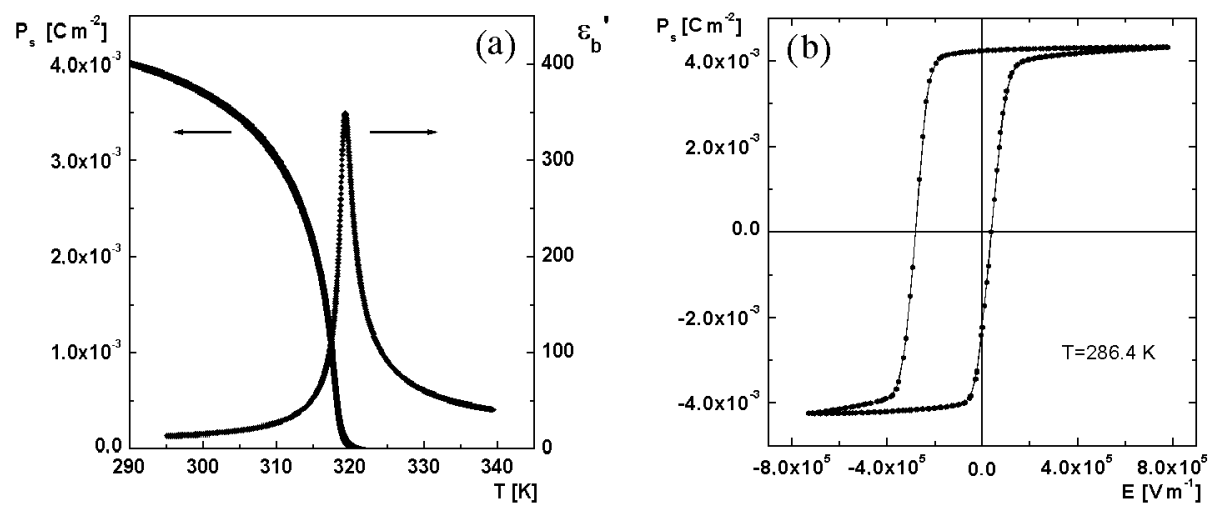

Fig. 1. (a) Temperature dependence of dielectric constant and spontaneous polarization for D-GPI; (b) hysteresis loop observed in D-GPI.

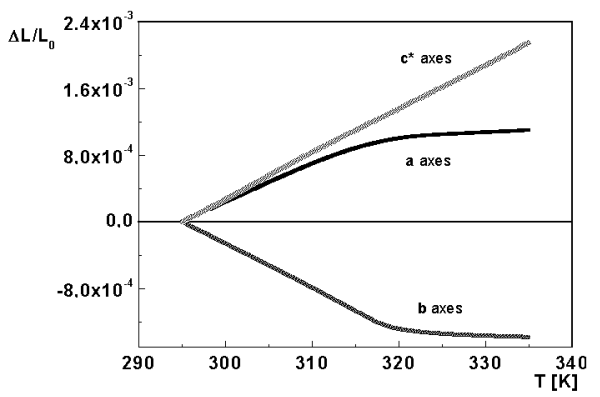

Fig. 2. Temperature dependence of relative expansion $\Delta L / L_{0}$.

contrary the change of relative expansion along the $b$-axis (ferroelectric one) diminishes. Round shape of this dependence is observed in the region of $T_{\mathrm{c}}$ and again linear and weak dependence appears. Relative expansion along the $c^{*}$-axis increases nearly linearly and no change of its character is observed at $T_{c}$. Diffused anomalies are most probably caused by $E_{\mathrm{b}}$ existence in the crystals.

Taking into account the changes of the sample lengths we could determine the relative change of crystal volume as a function of temperature. Temperature dependences of relative volume expansion $\Delta V / V_{0}\left(V_{0}\right.$ - volume of the sample at $273 \mathrm{~K}$ ) and volume expansion coefficient $\alpha_{V}$ as a function of temperature are illustrated in Fig. 3.

The temperature dependences of the quasilongitudinal $V_{1}(\boldsymbol{q}\|\boldsymbol{a}, \boldsymbol{e}\| \boldsymbol{a})$, $V_{3}\left(\boldsymbol{q}\left\|\boldsymbol{c}^{*}, \boldsymbol{e}\right\| \boldsymbol{c}^{*}\right)$ and pure longitudinal $V_{2}(\boldsymbol{q}\|\boldsymbol{b}, \boldsymbol{e}\| \boldsymbol{b})$ ultrasonic waves (USW) (here $q$ is the USW vector, $e$ is its polarization) in D-GPI crystals are presented in Fig. 4. The temperature dependences of the changes of USW have practically the same character in all three directions. We observed diffused anomalies of the ultrasonic velocities in the vicinity of the phase transition from the paraelectric 


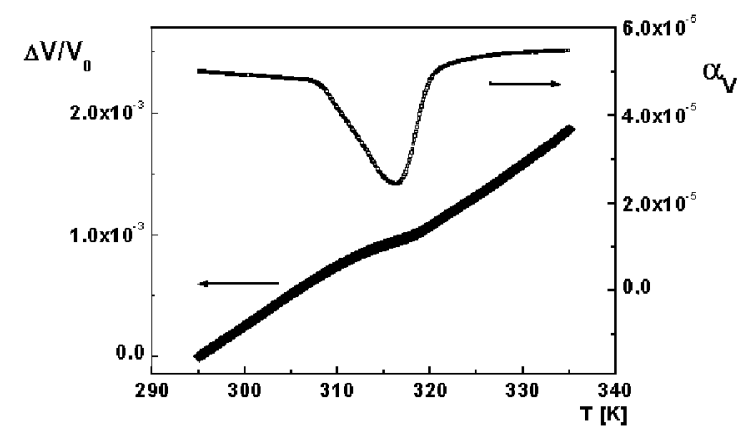

Fig. 3. Temperature dependence of relative volume expansion $\Delta V / V_{0}$ and expansion coefficient $\alpha_{V}$.

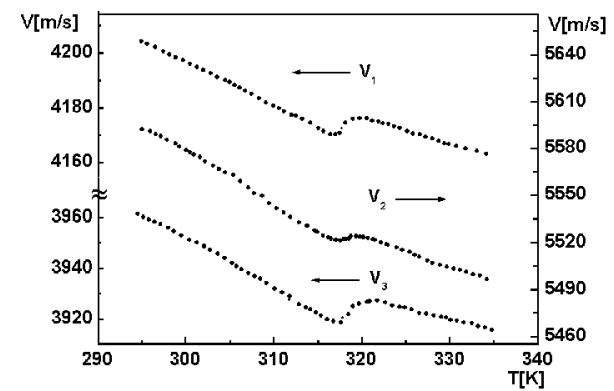

Fig. 4. The temperature dependence of the quasilongitudinal $V_{1}$ and $V_{3}$ and pure longitudinal $V_{2}$ USW velocities of D-GPI crystal.

to the ferroelectric phase. The changes of the USW velocities have been smaller than for the GPI crystal and round shape of the curves $i$ seen near the $T_{\mathrm{c}}$ is similar to the elongation measurements. The relative changes of the USW velocities $\Delta V_{i} / V_{i}(i=1,2,3)$ here amount to $0.18,0.09$, and $0.26 \%$ for the $a, b$, and $c^{*}$ directions, respectively. We did not observe any remarkable changes of USW velocities for the pure transverse $V_{4}\left(\boldsymbol{q}\|\boldsymbol{b}, \boldsymbol{e}\| \boldsymbol{c}^{*}\right)$ and quasitransverse $V_{5}\left(\boldsymbol{q}\left\|\boldsymbol{c}^{*}, \boldsymbol{e}\right\| \boldsymbol{a}\right)$ at $T=T_{\mathrm{c}}$. Only smooth decrease in the velocities with the change of temperature is observed as it is seen in Fig. 5. The changes of the pure transverse $V_{6}(\boldsymbol{q}\|\boldsymbol{b}, \boldsymbol{e}\| \boldsymbol{a})$ USW velocity is presented in Fig. 6 and a very small kink on its temperature dependence is seen at phase transition temperature. Generally, the ultrasonic results are quite similar to that we have obtained for the crystal GPI. The values of the thermal coefficients, which we have determined would not give any reasonable contribution to the changes of ultrasonic measurements so they were not considered in the temperature dependence of velocities. The explanation of the observed in experiment temperature dependences of USW velocities results is based on the Landau theory. Using the method described in [3] for GPI crystals we have written 


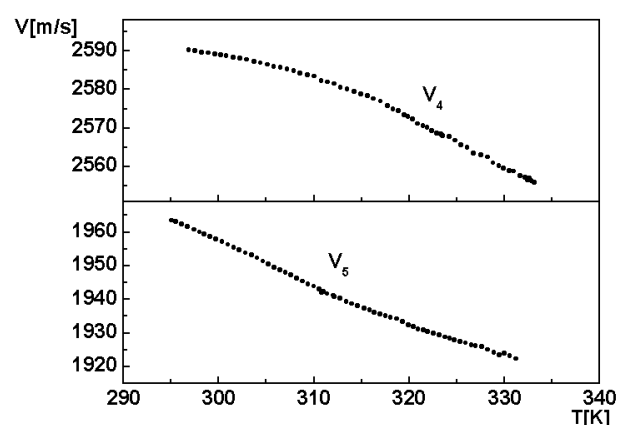

Fig. 5. The temperature dependence of the pure transverse $V_{4}$ and the quasitransverse $V_{5}$ USW velocity of D-GPI crystal.

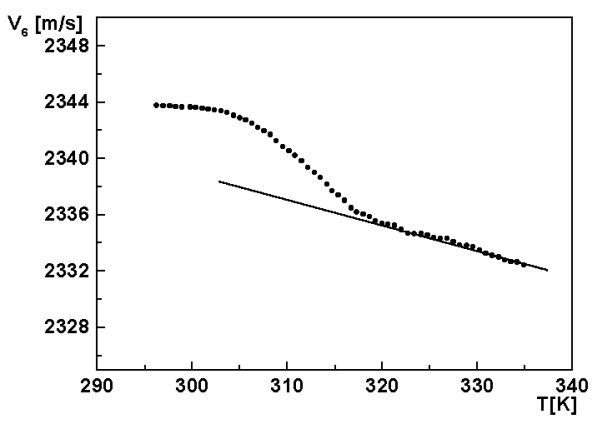

Fig. 6. The temperature dependence of the quasitransverse $V_{6}$ USW velocity of D-GPI crystal.

$$
\begin{aligned}
& \Delta C_{i j}^{*}=b_{i j} P_{0}^{2}-4 a_{i} a_{j} P_{0}^{2} /\left[\omega_{\mathrm{p}}^{2}(1+\mathrm{i} \Omega \tau)\right], \quad i, j=1,2,3,5, \\
& \Delta C_{44}^{*}=b_{44} P_{0}^{2}, \quad \Delta C_{66}^{*}=b_{66} P_{0}^{2}, \quad \Delta C_{46}^{*}=b_{46} P_{0}^{2},
\end{aligned}
$$

where $\Delta C_{i j}^{*}$ - elastic constant, $P_{0}^{2}=A\left(T_{\mathrm{c}}-T\right) / B$ is the equilibrium value of the spontaneous polarization, $\omega_{\mathrm{p}}^{2}=A\left(T-T_{\mathrm{c}}\right)$ - the soft mode frequency, $\Omega=2 \pi f$ - the USW frequency.

Comparing the experimental results presented in Fig. 6 with Cristofel's equations [3] and Eqs. (1a)-(1b) and assuming that $\Omega \tau \ll 1$, one can see a good qualitative agreement of the experiment and phenomenological theory. Particularly, as it follows from Cristofel's equations and Eq. (1a), the USW velocities $V_{1}, V_{2}, V_{3}$, and $V_{5}$ should exhibit a sudden decrease at $T_{\mathrm{c}}$, since they contain the contributions of the real part of the elastic constants which undergo the negative jumps $\left(\Delta C_{i j}=-2 a_{i} a_{j} / B\right.$, where $\left.i, j=1,2,3,5\right)$ at phase transition from para- to ferroelectric phase. The changes in those USW velocities below $T_{\mathrm{c}}$ are caused by the contribution of the first term of Eq. (1a), which appears as a result of the fourth-order anharmonic interaction between the order parameter and deformation ( $P^{2} U^{2}$-type coupling). This type of anharmonicity gives the contribution only into the real part leading to quadratic dependences of $V_{i}$ on the equilibrium value 
of spontaneous polarization at $T<T_{c}$. In the case of velocity $V_{5}$ such behavior is not observed, which is a very similar situation to pure crystal GPI where we have observed very small jump for that ultrasonic measurement. The behavior of the ultrasonic velocity $V_{4}$ is the example that in some direction the visible changes of parameters of ultrasonic waves near the phase transition temperature were not observed $[10,11]$. The anomalous temperature changes in the pure transverse USW $V_{6}$ below $T_{\mathrm{c}}$ are caused by the fourth-order anharmonicity only. The changes of the elastic constants are proportional to $P_{0}^{2}$, see Eq. (1b), which gives only the kinks on the $V_{6}(\mathrm{~T})$ temperature dependences at $T=T_{\mathrm{c}}$ (Fig. 6).

As the $T_{\mathrm{c}}$ point is not seen clearly in dilatometric and ultrasonic measurements we regard as the phase transition point the $T_{\mathrm{c}}$ found in dielectric measurement.

One can summarize the presented results as follows:

1. The existence of internal bias field is the characteristic feature of D-GPI crystal.

2. Dielectric constant and spontaneous polarization measurements confirmed the continuous character of phase transition.

3. Thermal expansion and ultrasonic velocities showed diffused anomalies in the vicinity of phase transition; it is probably attributed to the existence of internal bias field which influence the expansion and ultrasonic velocity.

\section{References}

[1] S. Dacko, Z. Czapla, J. Baran, M. Drozd, Phys. Lett. A 223, 2171 (1996).

[2] J. Baran, G. Bator, R. Jakubas, M. Śledź, J. Phys., Condens. Matter 8, 10647 (1966).

[3] J. Furtak, Z. Czapla, A.V. Kityk, Z. Naturforsch. A 52, 778 (1997).

[4] M.Th. Averbuch-Pouchot, Acta Crystallogr. C 49, 85 (1993).

[5] F. Shikanai, M. Komukae, Z. Czapla, T. Osaka, J. Phys. Soc. Jpn. 71, 498 (2002).

[6] B. Andrijewski, Z. Czapla, M. Romaniuk, O. Myshchyszyn, Phys. Status Solidi A 177, $575(2000)$.

[7] L. Guilbert, Z. Czapla, Appl. Opt. 40, 125 (2001).

[8] B. Kosturek, J. Baran, Ferroelectr. Lett. 27, 11 (2000).

[9] S. Dacko, Z. Czapla, Ferroelectr. Lett. 27, 17 (2000).

[10] M.J. Mehta, B.K. Basu, M.M. Raj, J. Phys. E, Sci. Instrum. 20, 1398 (1987).

[11] J. Furtak, Z. Czapla, A.V. Kityk, Ferroelectr. Lett. 22, 147 (1997). 